\title{
Ciphering and Deciphering Messages by Graph Labeling Techniques Through Multilevel Cryptosystem
}

\author{
B. Deepa, V. Maheswari
}

\begin{abstract}
In this paper we present an upgraded technique of coding a message using Multilevel Cryptosystem based on Four square Cipher, Mono alphabetic Substitution Cipher and Columnar Transposition cipher subjected to Graph labeling. We send our Cipher text in the form of graph pattern which admits Root cube mean labeling. Further by using the secret key the receiver decrypts the edge labels which is shared by the sender. Here we employ Symmetric key cryptosystem for both encryption and decryption of messages as both the sender as well as thereceiver shares the same key for encryption and decryption of messages. In this research work we performed Multilevel Cryptographic technique together with Graph Labeling concept to save the messages from third party or Hackers ensuring secure transmission of messages.
\end{abstract}

Keywords: Plaintext, Cipher text, Four Square cipher encryption, Mono alphabetic Substitution cipher and Columnar transposition cipher, Umbrella graph, Root Cube meanlabeling.

AMS Subject classification MSC (2010) No: $05 C 78$

\section{INTRODUCTION}

The R.Gowri,G.Vembarasi has presented the Wonderful notion of "Root cube mean labeling of Graphs" [1]and "Improved Advanced Encryption using Four Square Cipher for user anonymity and Un traceability in mobile cloud computing has been introduced by Solomon Babatunde and Shrikant Ojha [2] and "An Extended version of Four Square cipher using 10x10 Matrixes" has been introduced by J.Aishwarya, V.Palanisamy and K.Kanagaram [3] also from [4],[5] and [6] etc .So inspired by this work here we going to perform anupgraded technique of coding a message based on four square, mono alphabetic substitution and columnar transposition cipher for the graph $G$ using Root cube mean labeling. Earlier so many researchers have performed on the above ciphers but very rare on the Four square extended, so we are here proud to work on the continued type of foursquare ciphers with Graphic encryption, which continues to implement very easy and adaptable. So, we Present our cipher text in the form of graph pattern which is admits our proposed labeling technique. Further by using the secret key the receiver will decrypts the edge labels which are shared by sender. Here we performed Symmetric key for both encryption and decryption of messages.

Revised Manuscript Received on December 5, 2019.

B. Deepa, Research Scholar, Department of Mathematics, Vels Institute of Science, Technology \& Advanced Studies (VISTAS)Chennai-117, Tamilnadu-India

V. Maheswari,Associate Professor, Department of Mathematics, Vels Institute of Science, Technology \& Advanced Studies (VISTAS), Chennai117, Tamilnadu-India

\section{DEFINITIONS}

\section{A. Root Cube Mean Labeling}

A graph $G(V, E)$ with $p$ vertices and q edges is said to be a Root cube mean labeling if it is possible to label the vertices randomly with distinct elements then the function $\mathrm{f}$ from $0,1,2, \ldots \ldots q+1$ in such a way that each edge e is defined by uv and is denoted by

$f(e)=\sqrt{\frac{f(u)^{3}+f(v)^{3}}{2}}$. Here $\mathrm{f}$ is called as Root cube mean labeling.

\section{B. Four-Square Cipher}

In Cryptography, the Four-square cipher is also known as digraphic substitution cipher and it has manual symmetric encryption technique which means it can encrypt sets of letters which are exchanged by other sets of letters.

\section{Transposition Cipher}

In Cryptography, a transposition cipher is a technique of encryption by which the positions held by elements of plaintext are shifted according to a regular system, so that the ciphertext creates a transformation of the plaintext.

\section{Columnar Transposition}

As Columnar Transposition simply mixes the plaintext letter, it has to combined with any other stray cipher technique thereby making it difficult to perceive by any intruder.

\section{E. Cipher}

The message or information written in the form of code is itself known as a cipher.

\section{F. Clue}

A piece of indication giving a clue as to what is to be inserting in an exacting space of two vertices in our Cipher Graph to trace secret message shared by sender.

\section{G. Umbrella graph}

An umbrella graph $U(m, n)$ is the graph gained by joining a central vertex of $f_{m}$ with a path $p_{n}$.

\section{BASIC FOUR-SQUARE CIPHER}

Mr. Felix Delastelle was one of the well-known Cryptographer and he was invented a Four-square cipher in the year of 1840-1902.Also, his other famous inventions are Foursquare cipher, Bifid and Trifid Ciphers. For more details we refer to the description of Basic Four- Square Cipher discussed in [3]. 


\section{ENCRYPTION AND DECRYPTION TECHNIQUE}

STEP 1: Split up the message "attack at dawn" into two letters block i.e. "at ta ck at da wn" (Certain characters possibly attached to confirm the plaintext is an equivalent length).

STEP 2: To encrypt construct a bigram in the plain letters squares by utilize the two plain alphabet squares and two cipher alphabet squares in the area of four 'Squares'.

For Example, to encrypt 'at' in the plain letter's squares Transposition cipher is also one of the most important ciphers construct a bigram. The first and second letter placed in the top left and bottom left of squares respectively.

Table- I

\begin{tabular}{|c|c|c|c|c|c|c|c|c|c|}
\hline $\mathbf{a}$ & b & c & d & e & $\mathbf{z}$ & $\mathbf{G}$ & $\mathbf{P}$ & $\mathbf{T}$ & $\mathbf{F}$ \\
\hline$f$ & $g$ & h & $\mathbf{i}$ & $\mathbf{k}$ & o & $\mathbf{I}$ & $\mathbf{H}$ & $\mathbf{M}$ & $\mathbf{v}$ \\
\hline 1 & $\mathbf{m}$ & $\mathbf{n}$ & o & $\mathbf{p}$ & $\mathbf{w}$ & $\mathbf{D}$ & $\mathbf{R}$ & C & $\mathbf{N}$ \\
\hline $\mathbf{q}$ & $\mathbf{r}$ & s & $t$ & $\mathbf{u}$ & $\mathbf{Y}$ & $\mathbf{K}$ & $\mathbf{E}$ & $\mathbf{Q}$ & $\mathbf{A}$ \\
\hline $\mathrm{v}$ & $\mathbf{w}$ & $\mathbf{x}$ & $y$ & $z$ & $\mathbf{x}$ & $\mathbf{v}$ & $\mathbf{s}$ & B & $\mathbf{L}$ \\
\hline $\mathbf{M}$ & $\mathbf{F}$ & $\mathbf{N}$ & $\mathbf{B}$ & D & $\mathbf{a}$ & b & c & d & e \\
\hline C & $\mathbf{R}$ & $\mathbf{H}$ & $\mathbf{s}$ & $\mathbf{A}$ & f & $\mathbf{g}$ & $\mathbf{h}$ & $\mathbf{i}$ & $\mathbf{k}$ \\
\hline $\mathbf{x}$ & $\mathbf{Y}$ & 0 & G & $\mathbf{v}$ & 1 & $\mathrm{~m}$ & $\mathrm{n}$ & o & p \\
\hline $\mathbf{I}$ & $\mathbf{T}$ & $\mathbf{u}$ & $\mathbf{E}$ & $\mathbf{w}$ & $q$ & $\mathbf{r}$ & $s$ & $t$ & $\mathbf{u}$ \\
\hline $\mathbf{L}$ & Q & $z$ & $\mathbf{K}$ & $\mathbf{P}$ & $v$ & $w$ & $\mathbf{x}$ & $y$ & x \\
\hline
\end{tabular}

STEP 3: Positioning the letters in the ciphertext at the rectangle corners then the letter 'at' become.

Table- II

\begin{tabular}{|c|c|c|c|c|c|c|c|c|c|}
\hline$a_{A}$ & $b$ & $e$ & $-d-$ & e- & $z$ & $G$ & $P$ & $T$ & $\mathbf{F}$ \\
\hline $\mathbf{f}$ & g & h & i & $\mathbf{k}$ & o & I & H & $\mathrm{M}$ & $\mathbf{U}$ \\
\hline 1 & $\mathbf{m}$ & $\mathbf{n}$ & o & $\mathbf{p}$ & $\mathbf{w}$ & D & $\mathbf{R}$ & $c$ & $\mathbf{N}$ \\
\hline $\mathbf{q}$ & $\mathbf{r}$ & s & $t$ & $\mathbf{u}$ & $\mathbf{Y}$ & $\mathbf{K}$ & $\mathbf{E}$ & $\phi$ & $\mathbf{A}$ \\
\hline $\mathbf{v}$ & $w$ & $x$ & $y$ & $z$ & $\mathbf{x}$ & $\mathbf{v}$ & $\mathrm{S}$ & B & $\mathbf{L}$ \\
\hline$M$ & $\mathbf{F}$ & $\mathbf{N}$ & B & D & $\mathbf{a}$ & b & c & d & e \\
\hline C & $\mathbf{R}$ & H & $\mathbf{S}$ & $\mathbf{A}$ & f & g & h & $\mathrm{i}$ & $\mathbf{k}$ \\
\hline $\mathbf{x}$ & $\mathbf{Y}$ & o & G & $\mathbf{v}$ & 1 & $\mathbf{m}$ & $\mathbf{n}$ & o & $\mathbf{p}$ \\
\hline I & $\mathbf{T}$ & $\mathbf{U}$ & $\mathbf{E}$ & $\mathbf{w}$ & $\mathbf{q}$ & $\mathbf{r}$ & s & $t^{\nabla}$ & $\mathbf{u}$ \\
\hline $\mathbf{L}$ & Q & $\mathrm{Z}$ & $\mathbf{K}$ & $\mathbf{P}$ & $\mathbf{v}$ & $w$ & $\mathbf{x}$ & $y$ & z \\
\hline
\end{tabular}

STEP 4: By using the above keys, the bigram 'at' is coded into 'TI'

STEP 5: In a similar way the plaintext 'attack at dawn' is encrypted into TIYBFHTIZBSY

With the help of keys which are 'ZGPTFOIHMUWDRCNYKEQAXVSBL'

'MFNBDCRHSAXYOGVITUEWLQZKP'.

\section{MONOALPHABETIC SUBSTITUTION METHOD}

The history of monoalphabetic substitution cipher can be drawn back to the very initial civilisations, the monoalphabetic substitution cipher, also called as a simple substitution cipher, depend on a fixed alternative structure. That is, the replacement is fixed for each letter of the alphabet. Thus, if "A" is encrypted to "z", then every time we see the letter "A" in the plaintext, we replace it with the letter " $\mathrm{z}$ " in the ciphertext.

\section{TRANSPOSITION CIPHER}

in the classical cipher's family,In the Period of 2003 it is generally used in the construction field of modern block ciphers in that we are using transposition cipher combined with columnar transposition cipher.

The columnar transposition cipher is an easy simple cipher, flexible to implement. It is a transposition cipher that follows a simple rule for arrange up the letters in the plaintext to form the ciphertext. It can be combined with other ciphers, such as a Four-Square cipher and Monoalphabetic substitution cipher, the combination of which can be more difficult to break.

Flow Chart - Proposal for Multilevel Security

\section{ENCRYPTION PROCESS}

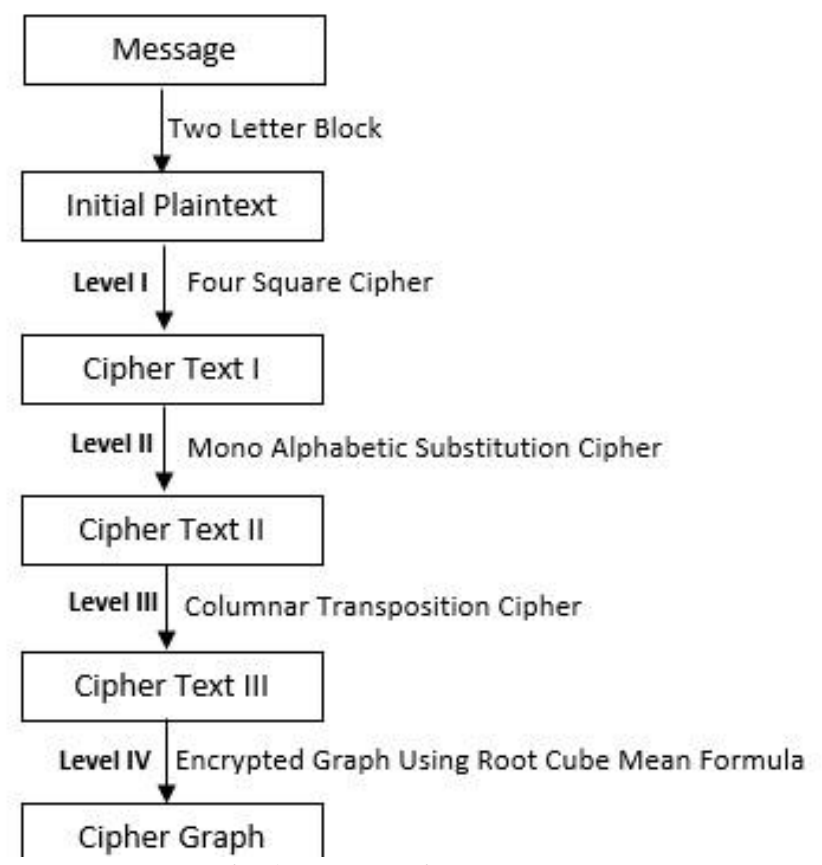

Fig.1. Encryption Process 


\section{DECRYPTION PROCESS}

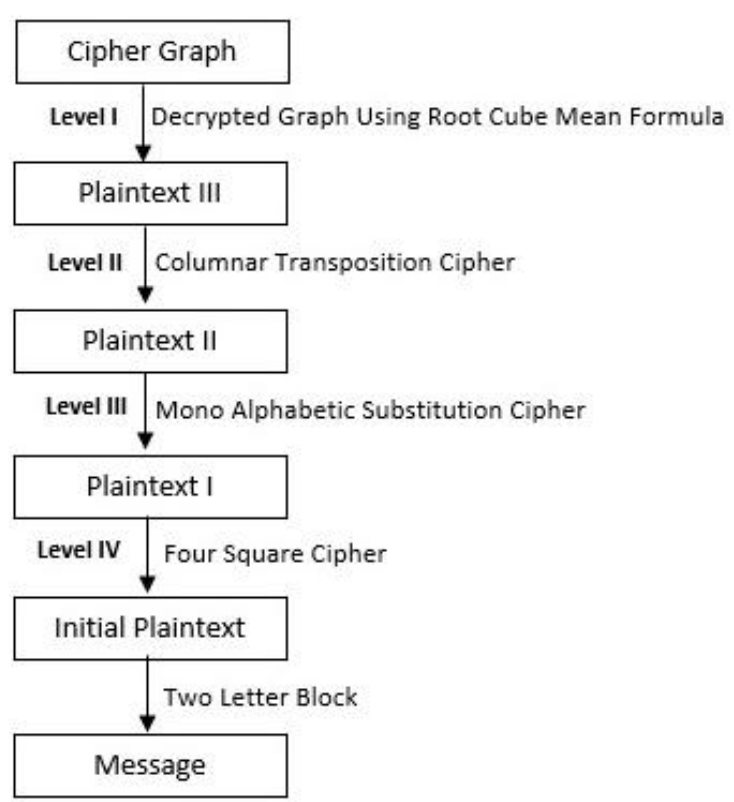

Fig.2. Decryption Process

\section{ILLUSTRATION I- OUR PROPOSED WORK}

\section{H. LEVEL I: Four Square Cipher}

Let our message be "there is 5 * on my car".

STEP 1: Our initial plaintext is obtained by Splitting up the message into two letters block as follows

Table III: Initial plaintext in two letter blocks

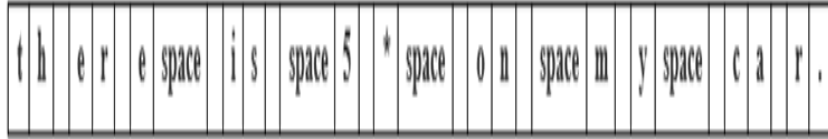

(Certain characters possibly attached to confirm the plaintext is an equivalent length)

STEP 2: Locate the beginning letter of the plaintext message appearing in the provided plaintext, placed at Top-left10 $\times$ 10 matrices.

Table-IV: $10 \times 10$ matrices

\begin{tabular}{|c|c|c|c|c|c|c|c|c|c|c|c|c|c|c|c|c|c|c|c|}
\hline & b & e & d & & & g & w & & & G & 0 & & $\mathrm{~N}$ & 3 & $\mathrm{~V}$ & 2 & $\equiv$ & & $\geq$ \\
\hline | & $\mathrm{n}$ & $\mathrm{n}$ & p & $q$ & $s$ & $\mathrm{u}$ & v & w & $x$ & 4 & & & 7 & 8 & $\mathrm{U}$ & w & $n$ & $\mathrm{U}$ & \\
\hline space & 2 & : & 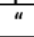 & ? & $>$ & $<$ & 0 & & + & 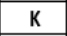 & $\mathrm{H}$ & 9 & & + & $\mathrm{T}$ & $x$ & D & $r$ & 1 \\
\hline$!$ & @ & $\#$ & $\$$ & $y$ & $\%$ & $\Lambda$ & 8 & 1 & . & $\mathrm{L}$ & & & \{ & & $R$ & 2 & $\div$ & $\sim$ & \\
\hline$D$ & 0 & 6 & $\mathrm{i}$ & V & $\mathrm{U}$ & $\mathrm{C}$ & ก & U & $\leq$ & 1 & $Y$ & ' & ; & $h$ & $Q$ & 0 & $M$ & $\mathrm{~J}$ & G \\
\hline$F$ & 1 & 7 & $\mathrm{~J}$ & $\mathrm{~K}$ & & $t$ & $\equiv$ & $r$ & 1 & $:$ & $s$ & $>$ & $<$ & A & B & C & $\mathrm{E}$ & $\mathrm{F}$ & 1 \\
\hline G & 2 & 8 & W & $\mathrm{L}$ & $S$ & $\geq$ & $\div$ & $\sim$ & & $!$ & @ & $\#$ & $\$$ & $y$ & $\%$ & n & $\&$ & 1 & \\
\hline T & 3 & 9 & $x$ & $M$ & $Q$ & \pm & 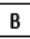 & 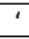 & ; & 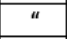 & : & $\mathrm{m}$ & $\mathrm{j}$ & $z$ & $y$ & $n$ & $x$ & $w$ & $v$ \\
\hline $\mathrm{H}$ & 4 & $Y$ & 1 & $\mathrm{~N}$ & 0 & $*$ & $=$ & A & - & $\mathrm{P}$ & $\mathrm{u}$ & $t$ & $r$ & $q$ & $p$ & 0 & $\mathrm{~m}$ & $k$ & \\
\hline I & 5 & Z & [ & $P$ & E & $R$ & 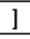 & \{ & \} & oace & $\mathrm{i}$ & g & 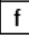 & 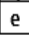 & - & b & $r$ & 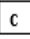 & d \\
\hline a & b & e & d & f & & & h & i & 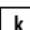 & G & & & $\mathrm{N}$ & 3 & $y$ & 2 & $=$ & & \\
\hline I & $\mathrm{n}$ & $n$ & $p$ & $q$ & $s$ & u & v & w & $\mathrm{x}$ & 4 & 5 & 6 & 1 & 8 & U & W & $n$ & U & $\leq$ \\
\hline space & $z$ & : & " & ? & $>$ & $<$ & 0 & & + & K & $\mathrm{H}$ & 9 & & + & $\mathrm{T}$ & $x$ & D & $r$ & 1 \\
\hline 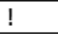 & @ & $\#$ & $\$$ & $y$ & $\%$ & $\Lambda$ & $\&$ & 1 & 1 & $\mathrm{~L}$ & [ & ] & \{ & \} & $\begin{array}{ll} \\
\end{array}$ & $\mathrm{Z}$ & $\div$ & $\sim$ & \\
\hline D & 0 & 6 & $i$ & $\mathrm{~V}$ & U & C & $\cap$ & U & - & 1 & $Y$ & 1 & ; & $h$ & Q & 0 & $M$ & $\mathrm{~J}$ & G \\
\hline$F$ & 1 & 7 & $\mathrm{~J}$ & $\mathrm{~K}$ & . & $t$ & $\equiv$ & - & 1 & 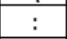 & $s$ & $>$ & $<$ & A & B & C & $\mathrm{E}$ & $\mathrm{F}$ & \\
\hline G & 2 & 8 & u & L & $S$ & $\geq$ & $\div$ & - & & $!$ & $@$ & $\#$ & $\$$ & $y$ & $\%$ & $A$ & $\&$ & 1 & \\
\hline $\mathrm{T}$ & 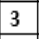 & 9 & $\mathrm{X}$ & $M$ & $Q$ & \pm & B & 4 & & " & $?$ & $\mathrm{~m}$ & 1 & 2 & $y$ & $n$ & $x$ & $w$ & v \\
\hline $\mathrm{H}$ & 4 & 1 & 1 & $\mathrm{~N}$ & 0 & 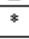 & $=$ & A & - & $\mathrm{p}$ & $\mathrm{u}$ & $t$ & $r$ & $q$ & $p$ & 0 & $\mathrm{~m}$ & k & \\
\hline 1 & 5 & Z & [ & $P$ & 5 & $\mathbf{R}$ & & & & space & i & $\mathrm{g}$ & $f$ & e & $a$ & b & & C & \\
\hline
\end{tabular}

STEP 3: Locate the associated second letter of the plaintext message appearing in the provided digraph placed at lowerright $10 \times 10$ plain text matrixes.
Table- V: $10 \times 10$ matrices

\begin{tabular}{|c|c|c|c|c|c|c|c|c|c|c|c|c|c|c|c|c|c|c|c|}
\hline 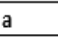 & b & e & d & $f$ & c & $\mathrm{g}$ & $\mathbf{h}$ & j & & $\mathrm{G}$ & 0 & 1 & $\mathbf{N}$ & 3 & $\mathrm{~V}$ & 2 & $\equiv$ & & \\
\hline I & $n$ & $\mathrm{n}$ & $\mathrm{p}$ & $q$ & $s$ & $\mathrm{u}$ & $v$ & $w$ & & 4 & & 6 & 7 & 8 & $\mathrm{U}$ & w & ก & $\mathrm{J}$ & \\
\hline space & & : & " & $?$ & $>$ & $<$ & 0 & & & $\mathrm{~K}$ & $\mathrm{H}$ & 9 & & + & $\mathrm{T}$ & $x$ & D & $r$ & 1 \\
\hline ! & @ & $\#$ & $\$$ & $y$ & $\%$ & ^ & $\&$ & 1 & & L & & ] & \{ & f & $R$ & Z & $\div$ & & \\
\hline D & 0 & 6 & $\mathbf{i}$ & $\mathrm{v}$ & $\mathbf{U}$ & $\mathrm{C}$ & $n$ & U & $\leq$ & 1 & $Y$ & ' & ; & h & $Q$ & 0 & $M$ & J & \\
\hline $\mathrm{F}$ & 1 & 7 & $J$ & $\mathrm{~K}$ & 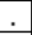 & $t$ & $\equiv$ & $r$ & f & $:$ & $s$ & $>$ & $<$ & A & B & C & $\mathrm{E}$ & $F$ & I \\
\hline G & 2 & 8 & $W$ & $\mathrm{~L}$ & $S$ & $\geq$ & $\div$ & $\sim$ & & $!$ & @ & $\#$ & $\$$ & y & $\%$ & $\Lambda$ & $\&$ & & 1 \\
\hline$T$ & 3 & 9 & $X$ & $M$ & $Q$ & \pm & B & ' & & " & & $\mathrm{m}$ & $j$ & z & $y$ & $\mathrm{n}$ & $x$ & w & $v$ \\
\hline $\mathrm{H}$ & 4 & $Y$ & 1 & $\mathrm{~N}$ & 0 & $*$ & $=$ & A & - & $\mathbf{P}$ & " & $t$ & $r$ & $q$ & $p$ & 0 & $\mathrm{~m}$ & k & \\
\hline I & 5 & Z & {[} & $\mathbf{P}$ & $\mathrm{E}$ & $\mathbf{R}$ & ] & \{ & \} & space & 1 & $\mathrm{~g}$ & $f$ & e & $a$ & b & $r$ & & d \\
\hline & & & & & & & & & & & & & & & & & & & \\
\hline a & b & e & d & $\mathrm{f}$ & c & $\mathrm{g}$ & $\mathbf{h}$ & $\mathrm{j}$ & & G & 0 & 1 & $\mathbf{N}$ & 3 & V & 2 & $\equiv$ & & \\
\hline I & $\mathrm{m}$ & $\mathrm{n}$ & p & $q$ & $\mathrm{~s}$ & $\mathrm{u}$ & $v$ & w & $\mathrm{x}$ & 4 & 5 & 6 & 7 & 8 & U & w & $\cap$ & U & $\leq$ \\
\hline space & $z$ & $:$ & $\|$ & $?$ & $>$ & $<$ & 0 & & + & $\mathrm{K}$ & H & 9 & & + & $\mathrm{T}$ & $x$ & D & $r$ & 1 \\
\hline$!$ & $@$ & $\#$ & $\$$ & $y$ & $\%$ & $\Lambda$ & $\&$ & 1 & 1 & $\mathrm{~L}$ & [ & ] & \{ & \} & $\mathrm{R}$ & Z & $\div$ & $\sim$ & \\
\hline D & 0 & 6 & $\mathbf{i}$ & $\mathbf{v}$ & $\mathbf{U}$ & C & $\cap$ & U & $\leq$ & 1 & $Y$ & ' & ; & h & Q & 0 & $M$ & J & G \\
\hline$F$ & 1 & 7 & $\mathrm{~J}$ & $\mathrm{~K}$ &. & $t$ & $\equiv$ & $r$ & 1 & 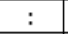 & $s$ & $>$ & $<$ & A & B & C & $E$ & $F$ & $I$ \\
\hline $\mathrm{G}$ & 2 & 8 & $W$ & $\mathrm{~L}$ & $S$ & $\geq$ & $\div$ & $\sim$ & & ! & @ & $\#$ & $\$$ & $y$ & $\%$ & $\Lambda$ & $\&$ & 1 & 1 \\
\hline$T$ & 3 & 9 & $\mathrm{x}$ & $\mathrm{M}$ & $Q$ & \pm & $B$ & ' & 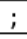 & “ & $?$ & $\mathrm{~m}$ & $\mathbf{j}$ & z & $y$ & $n$ & $\mathbf{x}$ & w & v \\
\hline $\mathrm{H}$ & 4 & $Y$ & 1 & $\mathrm{~N}$ & 0 & $*$ & $=$ & $\mathrm{A}$ & & p & $\mathrm{u}$ & $\mathrm{t}$ & $r$ & q & $p$ & 0 & $\mathrm{~m}$ & k & \\
\hline I & 5 & Z & ] & $\mathbf{P}$ & $\mathrm{E}$ & $\mathbf{R}$ & & & & space & & & & 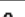 & & h & & c & \\
\hline
\end{tabular}

STEP 4: The encrypted digraph of the first letter is in the same row of the first plaintext letter and the same column of the second plaintext letter, hence therefore in the top-right cipher text matrix

STEP 5: The encrypted digraph of the Second letter is in the same column of the first plaintext letter and the same row of the second plaintext letter, hence therefore in the Lower-left cipher text matrix.

STEP 6: In a similar way our message "there is $5 *$ on my car." is encrypted into AC NY GZ YJ HI PR XB DH LP VE IA With the help of ciphertext squares.

The Encryption of "Four Square Cipher" simply consist of double letter corner of the rectangle.

Hence our two letters block of Initial plaintext becomes Ciphertext I

Ciphertext I:AC NY GZ YJ HI PR XB DH LP VE IA

Table- VI: $10 \times 10$ matrices

\begin{tabular}{|c|c|c|c|c|c|c|c|c|c|c|c|c|c|c|c|c|c|c|c|}
\hline$a$ & b & e & d & $\mathrm{f}$ & c & $\mathrm{g}$ & $\mathbf{h}$ & j & $\mathbf{k}$ & G & 0 & 1 & $\mathbf{N}$ & 3 & $\mathrm{v}$ & 2 & $\equiv$ & - & $\geq$ \\
\hline$I$ & $\mathrm{~m}$ & $n$ & $p$ & $q$ & $s$ & $\mathrm{u}$ & $\mathbf{v}$ & $w$ & $\mathbf{x}$ & 4 & 5 & 6 & \begin{tabular}{|l|}
7 \\
\end{tabular} & 8 & $\mathbf{U}$ & w & $\cap$ & $\mathrm{U}$ & $\leq$ \\
\hline space & $z$ & : & " & $?$ & $>$ & $<$ & 0 & & + & $\mathrm{K}$ & $\mathbf{H}$ & 9 & & + & $\mathbf{T}$ & $x$ & D & $r$ & 1 \\
\hline$!$ & $@$ & $\#$ & $\$$ & $y$ & $\%$ & $\wedge$ & $\&$ & 1 & 1 & $\mathrm{~L}$ & [ & ] & \{ & \} & $R$ & $\mathrm{Z}$ & $\div$ & $\sim$ & \\
\hline D & 0 & 6 & $\mathbf{i}$ & $\mathbf{v}$ & $\mathbf{u}$ & C & $\cap$ & U & $\leq$ & 1 & $\mathbf{Y}$ & ‘ & ; & h & $\mathbf{Q}$ & 0 & $\mathbf{M}$ & $\mathrm{J}$ & G \\
\hline$F$ & 1 & 7 & $\mathrm{~J}$ & $\mathbf{K}$ & . & $4-$ & $\equiv$ & $r$ & 1 & $\div$ & $s$ & $\rightarrow$ & $\leftrightarrow$ & $A_{1}$ & B & C & $E$ & $F$ & $I$ \\
\hline G & 2 & 8 & $\mathbf{w}$ & $\mathbf{L}$ & $\mathrm{S}$ & $\geq$ & $\div$ & \begin{tabular}{|l|} 
\\
\end{tabular} & ․ & $!$ & @ & $\#$ & $\$$ & $y$ & $\%$ & $\Lambda$ & $\&$ & 1 & 1 \\
\hline$T$ & 3 & 9 & $\mathrm{x}$ & $\mathbf{M}$ & $\mathbf{Q}$ & \pm & B & ${ }^{\prime}$ & ; & “ & $?$ & $\mathrm{~m}$ & $\mathbf{j}$ & $\mathrm{z}$ & $y$ & $n$ & $x$ & $\mathbf{w}$ & $v$ \\
\hline $\mathrm{H}$ & 4 & $\mathbf{Y}$ & 1 & $\mathbf{N}$ & 0 & $*$ & $=$ & A & - & $\mathbf{P}$ & $\mathrm{u}$ & $t$ & $r$ & $q$ & $p$ & 0 & $\mathrm{~m}$ & $k$ & . \\
\hline 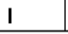 & 5 & Z & {[} & $\mathbf{P}$ & $\mathrm{E}$ & $R$ & ] & \{ & \} & space & $\mathbf{i}$ & $\mathrm{g}$ & $f$ & e & $\mathrm{a}$ & b & $r$ & \begin{tabular}{|l|l|} 
c \\
\end{tabular} & $d$ \\
\hline & & & & & & & & & & & & & & & & & & & \\
\hline a & b & e & d & $\mathrm{f}$ & $\mathbf{c}$ & $\mathrm{g}$ & $\mathbf{h}$ & $\mathbf{j}$ & $\mathbf{k}$ & G & 0 & 1 & $\mathbf{N}$ & 3 & $\mathrm{v}$ & 2 & $\equiv$ & - & $\geq$ \\
\hline 1 & $\mathrm{~m}$ & $n$ & $p$ & $q$ & $s$ & $\mathrm{u}$ & $\mathbf{v}$ & $w$ & $\mathrm{x}$ & 4 & 5 & 6 & 7 & 8 & $\mathbf{U}$ & w & $\cap$ & $\mathrm{U}$ & $\leq$ \\
\hline space & $z$ & $:$ & “ & $?$ & $>$ & $<$ & 0 & & + & K & $\mathrm{H}$ & 9 & & + & $\mathrm{T}$ & $x$ & D & $r$ & 1 \\
\hline$!$ & $@$ & $\#$ & $\$$ & $y$ & $\%$ & $n$ & $\&$ & 1 & 1 & $\mathrm{~L}$ & [ & ] & \{ & \} & $R$ & $z$ & $\div$ & $\sim$ & 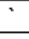 \\
\hline D & 0 & 6 & $\mathbf{i}$ & $\mathrm{v}$ & $\mathbf{U}$ & $c_{4}$ & $\cap$ & $U$ & $\leq$ & 1 & $y$ & 1 & i & $-h^{v}$ & $\mathbf{Q}$ & 0 & $\mathbf{M}$ & $\mathrm{J}$ & $\mathrm{G}$ \\
\hline$F$ & 1 & 7 & $\mathrm{~J}$ & $\mathbf{K}$ & . & $t$ & $\equiv$ & $r$ & 1 & : & $s$ & $>$ & $<$ & A & B & C & $\mathrm{E}$ & $F$ & I \\
\hline G & 2 & 8 & $\mathbf{w}$ & $\mathbf{L}$ & $\mathrm{S}$ & $\geq$ & $\div$ & $\sim$ & “ & $!$ & $@$ & $\#$ & $\$$ & $y$ & $\%$ & $\Lambda$ & $\&$ & 1 & 1 \\
\hline$T$ & 3 & 9 & $\mathrm{x}$ & $\mathrm{M}$ & $\mathbf{Q}$ & \pm & B & 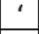 & ; & " & $?$ & $\mathrm{~m}$ & $\mathbf{j}$ & $z$ & $y$ & $n$ & $x$ & $w$ & $v$ \\
\hline H & 4 & $\mathbf{Y}$ & 1 & $\mathbf{N}$ & 0 & ${ }^{*}$ & $=$ & A & - & $\mathbf{P}$ & $\mathbf{u}$ & $t$ & $r$ & $q$ & $p$ & 0 & $\mathbf{m}$ & $\mathbf{k}$ & \\
\hline I & 5 & Z & [ & $\mathbf{P}$ & $E$ & $\mathbf{R}$ & ] & \{ & \} & space & $i$ & $\mathrm{~g}$ & $\mathrm{f}$ & $\mathrm{e}$ & $\mathrm{a}$ & b & $r$ & c & d \\
\hline
\end{tabular}

\section{VIII.LEVEL II: MONOALPHABETIC SUBSTITUTION METHOD}

The ciphertext I from Level I becomes our Plaintext I. This Plaintext I is now processed to Level II. At the end of Level 
II, the cipher text I becomes the plaintext II for level III In Monoalphabetic Substitution Cipher each letter is substituted by some other fixed letter of our choice. In our except the null values filled in the spare spaces for send our proposed method we just simply reverse the alphabetic order message in the form of graph as per Table 10. as per Table 7.

Table VII: Monoalphabetic Substitution Cipher

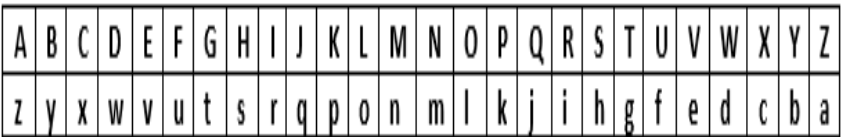

Plaintext I: AC NY GZ YJ HI PR XB DH LP VE IA

Ciphertext II: zX mb ta bqsrki cy ws ok evrz

\section{LEVEL III: TRANSPOSITION CIPHER}

The ciphertext II from Level II becomes our Plaintext II. This Plaintext II is now processed into Level III. To encrypt a fragment of text of our plaintext II, it has been filled in the columnar transposition table as follows. The key for the columnar transposition cipher is a keyword i.e. SOLVE. The row length that is used is the same as the length of the keyword.

Table: VIII

\begin{tabular}{|l|l|l|l|l|}
\hline S & O & L & V & E \\
\hline z & x & m & b & t \\
\hline $\mathbf{a}$ & $\mathbf{b}$ & $\mathbf{q}$ & s & r \\
\hline k & i & c & y & w \\
\hline s & o & $\mathbf{k}$ & e & v \\
\hline r & z & $\mathbf{l}$ & $\mathbf{2}$ & $\mathbf{3}$ \\
\hline
\end{tabular}

In our regular columnar transposition cipher, the additional spaces are filled with nulls i.e. 123 In that our plaintext has been filled so that it neatly fits in a rectangle. The columns are now reordered such that the letters in the key word are ordered alphabetically reversed.

Table: IX

\begin{tabular}{|l|l|l|l|l|}
\hline E & V & L & O & S \\
\hline t & b & m & $\mathbf{x}$ & z \\
\hline r & s & q & b & a \\
\hline w & y & c & i & k \\
\hline v & e & k & o & s \\
\hline 3 & 2 & l & z & r \\
\hline
\end{tabular}

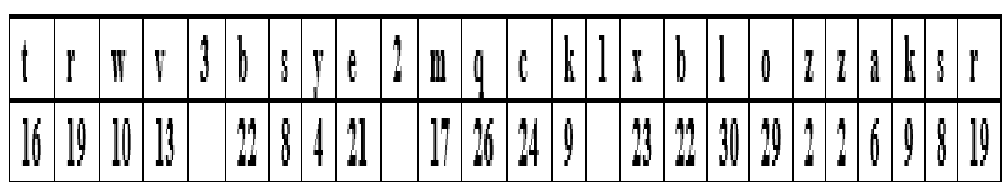

\section{LEVEL IV: ROOT CUBE MEAN LABELING}

The ciphertext III from Level III becomes our Plaintext III. This Plaintext III is now processed into Level IV.

Finally, our encrypted plaintext III presented in the form of Graph using Root Cube meanlabeling.

\section{Cipher Graph}

Cipher Graph is representing our Plaintext III Which is send to the receiver along with clue that are used by the sender. From which the receiver discovers the Edge labels by using Root Cube mean labeling. Its a primary step to trace our Message. We have taken Umbrella Graph to send our message more integrity and complexity.

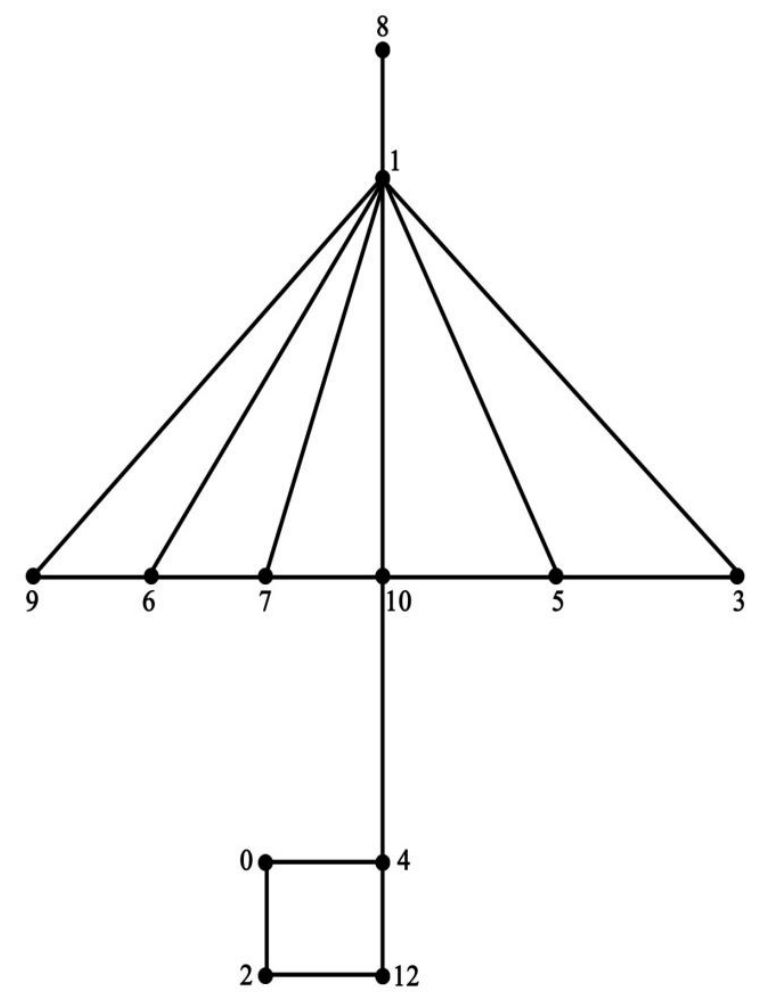

Fig:3- U(m, n)

\section{J. CLUE}

Key 1: Use Root Cube Mean formula to find the labels by using the formula $\sqrt{\frac{f(u)^{3}+f(v)^{3}}{2}}$ further the receiver has to assign the labels in the five blocks as per given below

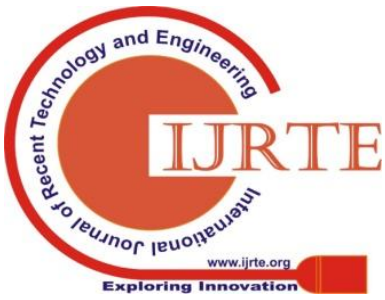




\begin{tabular}{|l|l|l|l|l|}
\hline \multicolumn{5}{|c|}{$\mathrm{E}$} \\
\hline $\boldsymbol{V}(8,1)$ & $\boldsymbol{V}(1,9)$ & $\boldsymbol{V}(1,6)$ & $\boldsymbol{V}(1,7)$ & \\
\hline $\mathbf{t}$ & $\mathbf{r}$ & $\mathbf{w}$ & $\mathrm{V}$ & $\mathbf{3}$ \\
\hline
\end{tabular}

\begin{tabular}{|l|l|l|l|l|}
\hline \multicolumn{5}{|c|}{$\mathrm{V}$} \\
\hline$V(1,10)$ & $V(1,5)$ & $V(1,3)$ & $V(9,6)$ & \\
\hline 22 & 8 & 4 & 21 & 2 \\
\hline
\end{tabular}

\begin{tabular}{|l|l|l|l|l|}
\hline \multicolumn{5}{|c|}{$\mathrm{L}$} \\
\hline$V(6,7)$ & $V(7,10)$ & $V(10,5)$ & $V(5,3)$ & \\
\hline 17 & 26 & 24 & 9 & 1 \\
\hline
\end{tabular}

\begin{tabular}{|l|l|l|l|l|}
\hline \multicolumn{5}{|c|}{ O } \\
\hline$V(10,4)$ & $V(1,10)$ & $V(4,12)$ & $V(12,2)$ & $V(2,0)$ \\
\hline 23 & 22 & 30 & 29 & 2 \\
\hline
\end{tabular}

\begin{tabular}{|c|l|l|l|l|}
\hline \multicolumn{5}{|c|}{$\mathrm{S}$} \\
\hline$V(2,0)$ & $V(0,6)$ & $V(5,3)$ & $V(1,5)$ & $V(1,9)$ \\
\hline 2 & 6 & 9 & 8 & 19 \\
\hline
\end{tabular}

Key 2: The key for the columnar transposition cipher is SOLVE.

Key3: The key for the Monoalphabetic Substitution Cipher simply reverse the alphabetic order.

\section{DECRYPTION PROCESS}

The receiver has to use the key formula of root cube mean labeling for finding the value of edges. Root cube mean formula is given by $\sqrt{\frac{f(u)^{3}+f(v)^{3}}{2}}$ which gives the following edges to trace our initial plaintext.

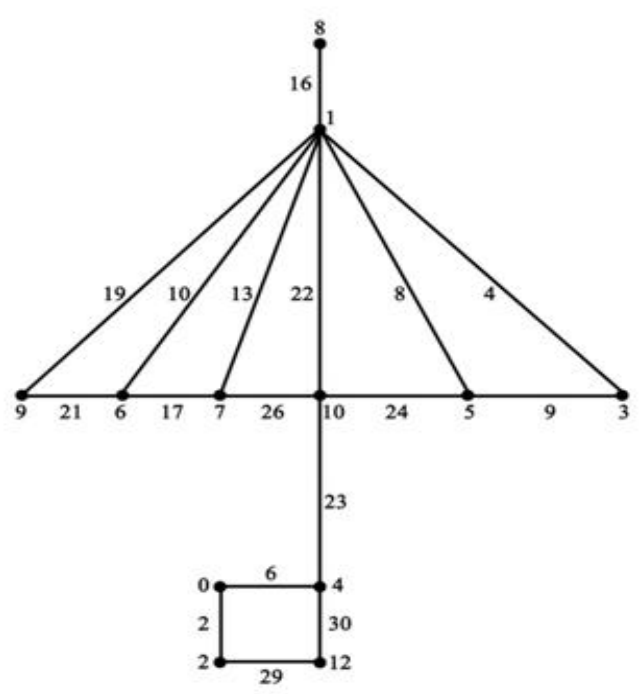

Fig:4- U(m, n)

\section{LEVEL I: TRANSPOSITION CIPHER}

From the above graph we are taking the edge value in the order of keyword blocks as shared by the sender. Further we are assigning letters for the edge labels as follows.
The letters are arranged in column wise with identical width corresponding to its keyword which is shown below the table.

Table: XI

\begin{tabular}{|l|l|l|l|l|}
\hline S & O & L & V & E \\
\hline z & x & m & b & t \\
\hline a & b & q & s & r \\
\hline k & i & c & y & w \\
\hline s & o & k & e & v \\
\hline r & z & l & 2 & 3 \\
\hline
\end{tabular}

further our letters are arranged in row wise into two letter blocks.

Then, we get Plaintext I: zx mb ta bqsrki cy ws ok evrz which is our ciphertext I for our next level process. Thus, our Plaintext I is now processed into Level II.

\section{XIII.LEVELII:MONOALPHABETIC SUBSTITUTION METHOD}

In the Decryption process of monoalphabetic substitutionmethod, alphabetical letters are arranged in reverse order.

Table: XII

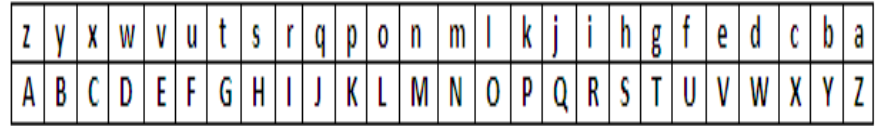

Taking corresponding values of "zx mb ta bqsrki cy ws ok evrz" from the above table. Then, our ciphertextlbecome our plaintext II.

Ciphertext I:zx mb ta bqsrki cy ws ok evrz

Plaintext II: AC NY GZ YJ HI PR XB DH LP VE IA

Thus, our Plaintext II is now processed into Level III which is taken as our ciphertext II in next level process.

\section{LEVEL III: FOUR SQUARE CIPHER}

Decryption of Four-Square Cipher works as the similar method, although in reversal. The cipher text II is the form of two letter blocks. The first letter moving in the Top-right hand matrix and the second letter moving in the low downleft hand matrix. Then new locations of the rectangle are then placed. These indicate the plaintext digraph. In this Top lefthand matrix component arriving in the beginning of our plaintext III.

\begin{tabular}{|c|c|c|c|c|c|c|c|c|c|c|c|c|c|c|c|c|c|c|c|c|c|c|c|c|}
\hline \multicolumn{5}{|c|}{$\mathbf{E}$} & \multicolumn{5}{|c|}{$\mathbf{V}$} & \multicolumn{5}{|c|}{ L } & \multicolumn{5}{|c|}{ O } & \multicolumn{5}{|c|}{$\mathbf{S}$} \\
\hline 16 & 19 & 10 & 13 & & 22 & 8 & 4 & 21 & & 17 & 26 & 24 & 9 & & 23 & 22 & 30 & 29 & 2 & 2 & 6 & 9 & 8 & 19 \\
\hline$t$ & $\mathbf{r}$ & $\mathbf{w}$ & $\mathbf{v}$ & 3 & b & $s$ & $\mathbf{y}$ & e & 2 & $\mathbf{m}$ & $q$ & c & $\mathbf{k}$ & 1 & $\mathbf{x}$ & b & 1 & 0 & $\mathbf{z}$ & $\mathbf{z}$ & $\mathbf{a}$ & $\mathbf{k}$ & $s$ & $\mathbf{r}$ \\
\hline
\end{tabular}


Table: XIII

\begin{tabular}{|c|c|c|c|c|c|c|c|c|c|c|c|c|c|c|c|c|c|c|c|}
\hline 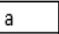 & $b$ & e & d & & & $g$ & $\mathrm{n}$ & & k & 0 & 0 & & $\mathrm{~N}$ & & V & 2 & $\equiv$ & - & $\geq$ \\
\hline . & & $n$ & $p$ & $q$ & & u & $\mathrm{v}$ & w & & 4 & 5 & & 7 & & U & W & $n$ & U & $\leq$ \\
\hline space & & : & 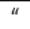 & ? & & $<$ & 0 & & + & K & $\mathrm{H}$ & 9 & & + & $T$ & $x$ & D & $r$ & / \\
\hline ! & @ & \# & $\$$ & $y$ & $\%$ & $\wedge$ & $\&$ & 1 & 1 & L & [ & ] & \{ & & $R$ & Z & $\div$ & $\sim$ & \\
\hline D & 0 & 6 & i & V & U & C & $n$ & $U$ & $\leq$ & 1 & $Y$ & ' & ; & $\mathrm{h}$ & $Q$ & 0 & $M$ & J & G \\
\hline $\mathrm{F}$ & 1 & 7 & $\mathrm{~J}$ & K & & $t$ & $\equiv$ & $r$ & 1 & : & 5 & $>$ & $<$ & A & $B$ & C & $E$ & $\mathrm{~F}$ & 1 \\
\hline G & 2 & 8 & W & L & S & $\geq$ & $\div$ & $\sim$ & , & $!$ & @ & \# & $\$$ & y & $\%$ & A & \& & 1 & 1 \\
\hline$T$ & 3 & 9 & $x$ & M & Q & \pm & $B$ & ' & ; & “ & ? & $\mathrm{m}$ & $j$ & $z$ & $y$ & $\mathrm{n}$ & $x$ & w & $\mathrm{v}$ \\
\hline $\mathrm{H}$ & 4 & $Y$ & 1 & $\mathrm{~N}$ & c & $*$ & $=$ & A & - & $P$ & u & $t$ & $r$ & q & $p$ & 0 & $\mathrm{~m}$ & $k$ & \\
\hline I & $J$ & Z & {[} & $P$ & $E$ & $R$ & ] & \{ & \} & oace & $i$ & g & $f$ & & a & b & $r$ & c & d \\
\hline & & & & & & & & & & & & & & & & & & & \\
\hline a & b & e & $d$ & $f$ & c & $\mathrm{g}$ & $\mathrm{h}$ & $j$ & $\mathrm{~K}$ & G & 0 & 1 & $N$ & 3 & V & 2 & $\equiv$ & - & $\geq$ \\
\hline I & $\mathrm{m}$ & $\mathrm{n}$ & $p$ & q & 5 & u & v & w & $\mathrm{x}$ & 4 & 5 & 6 & 7 & 8 & U & W & $n$ & U & \\
\hline space & $z$ & $:$ & " & $?$ & $>$ & $<$ & 0 & & + & K & $\mathrm{H}$ & 9 & 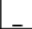 & + & $T$ & $x$ & D & $r$ & \\
\hline$!$ & @ & $\#$ & $\$$ & $y$ & $\%$ & $\wedge$ & $\&$ & 1 & 1 & $\mathrm{~L}$ & {[} & ] & 1 & \} & $R$ & $z$ & $\div$ & $\sim$ & \\
\hline D & 0 & 6 & $i$ & V & U & C & $n$ & U & $\leq$ & 1 & $Y$ & ' & ; & h & $Q$ & 0 & $M$ & J & G \\
\hline F & 1 & 7 & $\mathrm{~J}$ & K & . & $t$ & $\equiv$ & $r$ & 1 & : & 5 & $>$ & $<$ & A & $B$ & C & $E$ & $\mathrm{~F}$ & 1 \\
\hline $\mathrm{G}$ & 2 & 8 & W & $\mathrm{L}$ & $S$ & $\geq$ & $\div$ & $\sim$ & & $!$ & @ & \# & $\$$ & & $\%$ & $\Lambda$ & \& & & \\
\hline
\end{tabular}

Plaintext III is our final message I.e. There is $5^{*}$ on my car.

\section{ILLUSTRATION II}

\section{K. Level I: Four Square Cipher}

Let our message be "I see a bee!"

By splitting our message into two letter blocks we get,

Table: XIX

\begin{tabular}{|l|l|l|l|l|l|l|l|l|l|l|l|l|l|l|}
\hline I & space & & s & e & & e & space & & a & space & & b & e & \\
\hline
\end{tabular}

By using the Concepts of "Four Square Cipher" will give us Ciphertext I.

Table:XV- Four Square Cipher

\begin{tabular}{|c|c|c|c|c|c|c|c|c|c|c|c|c|c|c|c|c|c|c|c|}
\hline D & b & $\mathrm{V}$ & d & $f$ & $\mathrm{c}$ & $\mathrm{g}$ & $\mathrm{h}$ & $j$ & $\mathrm{k}$ & space & 0 & 1 & $\mathrm{~N}$ & $\mathrm{~W}$ & $\mathrm{~V}$ & 2 & $\equiv$ & - & $\geq$ \\
\hline 1 & $\mathrm{~m}$ & $\mathrm{e}$ & p & $q$ & $s$ & , & $\mathbf{v}$ & $w$ & $x$ & $\mathrm{~K}$ & 5 & 6 & 7 & $\mathrm{U}$ & 8 & 3 & n & u & $\leq$ \\
\hline space & z & : & " & ? & $>$ & $<$ & 0 & 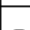 & + & 4 & $\mathrm{H}$ & 9 & 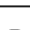 & + & $\mathrm{T}$ & $\mathrm{X}$ & D & $\begin{array}{ll}r \\
\end{array}$ & I \\
\hline$!$ & @a & $\#$ & $\$$ & $y$ & $\%$ & $\wedge$ & \& & ( & ) & L & [ & ] & \{ & \} & R & $\mathrm{Z}$ & $\div$ & $\sim$ & 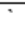 \\
\hline $\mathbf{a}$ & 0 & 6 & i & $\mathrm{n}$ & $\mathrm{U}$ & C & $n$ & u & $=$ & $\mathrm{Y}$ & & 6 & ; & h & Q & 0 & M & $\mathrm{J}$ & $\mathrm{G}$ \\
\hline$F$ & 1 & 7 & $\mathrm{~J}$ & $\mathrm{~K}$ & . & $\mathrm{t}$ & $\equiv$ & $\mathrm{r}$ & $f$ & : & $\mathrm{s}$ & $>$ & $<$ & $A$ & B & $\mathrm{C}$ & $E$ & $\mathrm{~F}$ & I \\
\hline G & 2 & $\mathrm{X}$ & W & L & $\mathrm{S}$ & $\geq$ & $\div$ & $\sim \sim$ & & $!$ & (a) & \# & $\$$ & $y$ & $\%$ & $\wedge$ & $\&$ & ( & ) \\
\hline $\mathrm{T}$ & 3 & 9 & 8 & M & Q & \pm & B & 6 & ; & $"$ & $?$ & $\mathrm{~m}$ & $j$ & $\mathrm{z}$ & $y$ & $\mathrm{n}$ & $x$ & $w$ & $\mathrm{v}$ \\
\hline $\mathrm{H}$ & 4 & $Y$ & $T$ & $\mathbf{N}$ & 0 & * & $=$ & A & - & $\mathrm{P}$ & $\mathbf{u}$ & $t$ & $\mathbf{r}$ & $q$ & $\mathrm{p}$ & 0 & $\mathrm{~m}$ & $\mathrm{k}$ & . \\
\hline I & $\mathrm{Z}$ & 5 & [ & $P$ & $\mathrm{R}$ & $E$ & ] & \{ & \} & $\mathrm{G}$ & $\mathrm{i}$ & $\mathrm{g}$ & $f$ & $\mathrm{e}$ & $\mathbf{a}$ & b & $\mathbf{r}$ & c & d \\
\hline D & b & $\mathrm{V}$ & d & f & c & $\mathrm{g}$ & h & J & $\mathrm{k}$ & \begin{tabular}{|l|} 
space \\
\end{tabular} & 0 & l & $\mathrm{N}$ & $\mathrm{W}$ & $\mathrm{V}$ & 2 & $\equiv$ & - & $\geq$ \\
\hline 1 & $\mathrm{~m}$ & e & $p$ & $q$ & $\mathrm{~s}$ & u & $\mathbf{v}$ & w & $x$ & $\mathrm{~K}$ & 5 & 6 & 7 & $\mathrm{U}$ & 8 & 3 & $n$ & u & $\leq$ \\
\hline space & $\mathrm{z}$ & : & " & $?$ & $>$ & $<$ & 0 & - & + & 4 & $\mathrm{H}$ & 9 & - & + & $\mathrm{T}$ & $\mathbf{X}$ & D & $\mathbf{r}$ & I \\
\hline$!$ & @ & $\#$ & $\$$ & $y$ & $\%$ & $\wedge$ & $\&$ & ( & ) & L & [ & ] & \{ & \} & $\mathrm{R}$ & $\mathrm{Z}$ & $\div$ & $\sim$ & \\
\hline $\mathbf{a}$ & 0 & 6 & $\mathbf{i}$ & $\mathrm{n}$ & $\mathrm{U}$ & $\mathrm{C}$ & $n$ & u & $\leq$ & $\mathrm{Y}$ & 1 & ' & ; & $\mathbf{h}$ & $Q$ & 0 & $\mathbf{M}$ & $\mathrm{J}$ & $\mathrm{G}$ \\
\hline $\mathrm{F}$ & 1 & 7 & $\mathbf{J}$ & $\mathrm{K}$ & . & $t$ & $\equiv$ & $\mathrm{r}$ & I & : & $\mathrm{s}$ & $>$ & $<$ & A & B & C & $\mathrm{E}$ & $\mathrm{F}$ & I \\
\hline G & 2 & $\mathrm{X}$ & W & L & $\mathrm{S}$ & $\geq$ & $\div$ & $\sim \sim$ & , & $!$ & (a) & \# & $\$$ & $y$ & $\%$ & $\Lambda$ & $\&$ & ( & ) \\
\hline $\mathrm{T}$ & 3 & 9 & 8 & M & Q & \pm & B & 6 & ; & " & $?$ & $\mathbf{m}$ & $j$ & 2 & $\mathrm{y}$ & $\mathrm{n}$ & $x$ & $w$ & $\mathrm{v}$ \\
\hline $\mathrm{H}$ & 4 & $\mathrm{Y}$ & 1 & $\mathrm{~N}$ & 0 & * & $=$ & $A$ & - & $P$ & $\mathrm{u}$ & $t$ & $r$ & $q$ & $\mathrm{P}$ & 0 & $\mathrm{~m}$ & $\mathrm{k}$ & . \\
\hline I & $\mathrm{Z}$ & 5 & [ & $P$ & $\mathbf{R}$ & $\bar{E}$ & 1 & \{ & \} & G & $\mathbf{i}$ & $\mathrm{g}$ & $f$ & $\mathrm{e}$ & $\mathbf{a}$ & b & $\mathbf{r}$ & c & d \\
\hline
\end{tabular}

Hence, our Ciphertext I: "GD UR KV YD WZ KX"

\section{LEVEL II: MONOALPHABETIC SUBSTITUTION METHOD}

The ciphertext I from Level I becomes our Plaintext I. This Plaintext I is now processed to Level II. At the end of Level II, the cipher text I becomes the plaintext II for level III.

By using the Concepts of "Monoalphabetic Substitution Method" will give us Ciphertext II.
Table- XVI

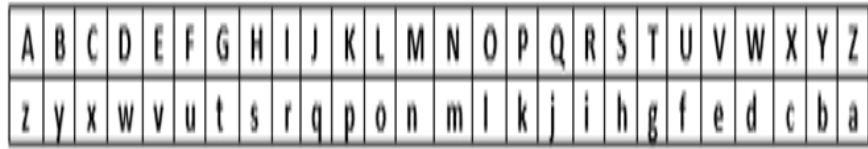

Hence, our Ciphertext I: "tw fi pe bw da pc"

\section{LEVEL III: TRANSPOSITION CIPHER}

The ciphertext II from Level II becomes our Plaintext II. This Plaintext II is now processed into Level III. To encrypt a fragment of text of our plaintext II,it has been filled in the columnar transposition table as follows. The keyword for our proposed method is "WORD".

Table- XVII

\begin{tabular}{|l|l|l|l|}
\hline $\mathbf{W}$ & $\mathbf{O}$ & $\mathbf{R}$ & $\mathbf{D}$ \\
\hline $\mathbf{t}$ & $\mathbf{w}$ & $\mathbf{f}$ & $\mathbf{i}$ \\
\hline $\mathbf{p}$ & $\mathbf{e}$ & $\mathbf{b}$ & $\mathbf{w}$ \\
\hline $\mathbf{d}$ & $\mathbf{a}$ & $\mathbf{p}$ & $\mathbf{c}$ \\
\hline
\end{tabular}

By using the Concepts of "Transposition Cipher" will give us Ciphertext III.

\begin{tabular}{|l|l|l|l|}
\multicolumn{5}{|c}{ Table- XVIII } \\
\hline $\mathbf{D}$ & $\mathbf{R}$ & $\mathbf{O}$ & $\mathbf{W}$ \\
\hline $\mathbf{i}$ & $\mathbf{f}$ & $\mathbf{w}$ & $\mathbf{t}$ \\
\hline $\mathbf{w}$ & $\mathbf{b}$ & $\mathbf{e}$ & $\mathbf{p}$ \\
\hline $\mathbf{c}$ & $\mathbf{p}$ & $\mathbf{a}$ & $\mathbf{d}$ \\
\hline
\end{tabular}

Hence our ciphertext III is written in the table below.

Table- XIX

\begin{tabular}{|l|l|l|l|l|l|l|l|l|l|l|l|}
\hline t & p & d & w & e & a & f & b & p & i & w & c \\
\hline
\end{tabular}

Finally, our ciphertext III is transformed into numerical value.

Table- XX

\begin{tabular}{|l|l|l|l|l|l|l|l|l|l|l|l|}
\hline t & p & d & w & e & a & f & b & p & i & w & c \\
\hline 13 & 15 & 14 & 12 & 14 & 7 & 4 & 2 & 16 & 2 & 6 & 17 \\
\hline
\end{tabular}

\section{XVIII.LEVEL IV: ROOT CUBE MEAN LABELING}

The ciphertext III from Level III becomes our Plaintext III. This Plaintext III is now processed into Level IV .

Finally, our encrypted plaintext III presented in the form of Graph using Root Cube mean labeling.

\section{XIX.CIPHER GRAPH}

Cipher Graph is representing our Plaintext III Which is send to the receiver along with clue that are used by the sender. From which the receiver discovers the Edge labels by using Root Cube mean 
labeling. It's a primary step to trace our Message.

We have taken Double Diamond Graph to send our message with more integrity and complexity.

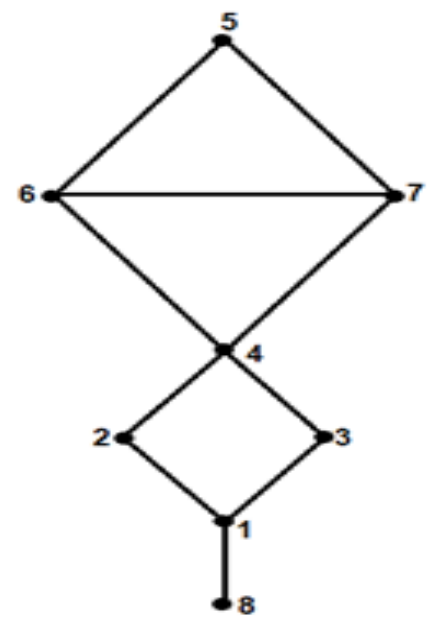

Fig :5- Double Diamond Graph

\section{CLUE}

Key 1: Use Root Cube Mean formula to find the labels by using the formula $\sqrt{\frac{f(u)^{3}+f(v)^{3}}{2}}$ further the receiver has to assign the labels in the five blocks as per given below.

\begin{tabular}{|c|c|c|}
\hline \multicolumn{3}{|c|}{$\mathrm{D}$} \\
\hline $\mathrm{t}$ & $\mathrm{P}$ & $\mathrm{d}$ \\
\hline$V(6,5)$ & $V(5,7)$ & $V(7,4)$ \\
& & \\
\hline \multicolumn{3}{|c|}{0} \\
\hline $\mathrm{f}$ & $\mathrm{b}$ & $\mathrm{p}$ \\
& & \\
\hline$V(3,1)$ & $V(2,1)$ & $V(1,18)$ \\
& & \\
\hline
\end{tabular}

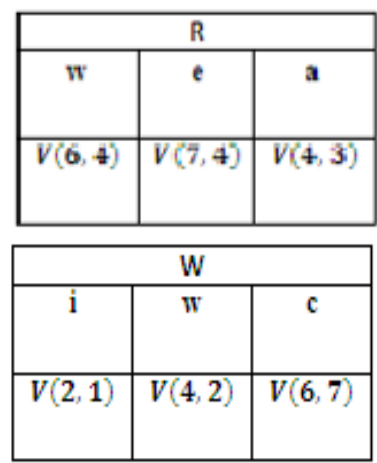

Key 2: The key for the columnar transposition cipher is "WORD".

Key3: The key for the Monoalphabetic Substitution Cipher simply reverse the alphabetic order.

\section{XX.DECRYPTION PROCESS}

The Decryption procedure is like Illustration I.

So, the process of Decryption at Multi level Cryptosystem will give us our Message

Hence our Message is "I see a bee!"

\section{XXI.CONCLUSION}

Thus, we have implemented a Multilevel Cryptosystem by making use of Four-Square Cipher, Monoalphabetic Substitution and Columnar Transposition technique for coding secret messages. The complexity of the system isfurther enhanced by combining it with Graph labeling which provides ultimate safety and security. For future Research in this area differentencryption techniques combined with any other kind of labeling can also be implemented.

\section{REFERENCES}

1. R. Gowri, G. Vembarasi "Root cube mean labeling of Graphs", International Journal of Engineering Science, Advanced Computing and Biotechnology, Vol.8, No.4, October - December 2017, pp.248255.

2. Solomon Babatunde Olaleye, Shrikant Ojha "Improved Advanced Encryption using Four Square Cipher for user anonymity and Untraceability in mobile cloud computing"-IJISET, Vol.4 Issue 2 , February 2017, ISSN(Online) 2348-7968/Impact Factor (2016)-5.264.

3. J. Aishwarya, V. Palanisamy and K. Kanagaram, "An Extended version of Four-Square cipher using 10x10 Matrixes "International Journal of Computer Applications (0975-8887); Volume 97-No.21, July 2014.

4. S. Kulandhai Therese, K. Romila, "Cube Root Cube Mean Labeling of Graphs" IJMTT-Volume 65 Issue 2-February 2019.

5. S. Sandhya, S. Somasundaram and S. Anusa, "Some More Results on Root Square Mean Graphs, Journal of Mathematics Research. \&, No.1;(2015), 72-81.

6. B. Deepa, Dr.V. Maheswari "Encoding and Decoding Using Graph Labeling", ISSN NO:0886-9367, IJAEM.

7. V.N.JayaShruthy, V. Maheswari, "Double Encryption, Decryption Process Using Graph Labeling Through Enhanced Vigenere Cipher", Journal of Physics: Conference Series, 2019.

8. Nihad Ahmad Hassan, Rami Hijazi. Introduction and Historical Background", Elsevier BV,2017

9. S. Sandhya, S. Somasundaram, and S. Anusa. "Degree Splitting of Root Square Mean Graphs", Applied Mathematics, 2 June 2015, 940 952.

\section{AUTHORS PROFILE}

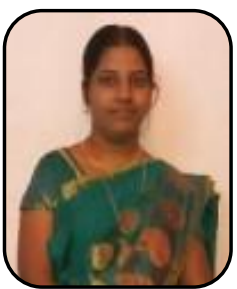

B. Deepa, Research Scholar, Department of Mathematics, Vels Institute of Science, Technology \&Advanced Studies (VISTAS), Pallavaram, Chennai-117, Tamilnadu, India. She continues her career in Sindhi Arts and Science College as an Assistant professor in Department of Mathematics since2013. And served as a Guest Lecture in Department of Mathematics at Indira Gandh Engineering College, Chengalpattu. Tamilnadu. Interesting area in Mathematics is Graph theory, Cryptography. She is now pursuing her Ph.D. in VISTAS ,Chennai. And she has published two research articles in in both National and International journals they are Encoding and Decoding using Graph Labeling and

Creating Ciphertext and Decipher using Graph Labeling Techniques.

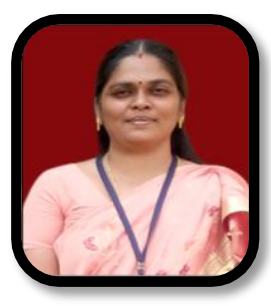

Dr.VMaheswari She is working at Vels Institute of Science, Technology\&AdvancedStudies (VISTAS) Chennai since 2017 to till date. She completedM.Sc., M.Phil., in Mathematics and her Ph.D. in (Graph Theory) Mathematics in ManoneManiyamSundranarUniversity, Tirunelveli. She has sixteen years of Teaching experience. Her research interests are Graph Labelings, Cryptography, she has published more than 15 research articles in both National and International journals. She has guided 3MPhil Scholars and is guiding 5Ph.D scholars. 